\title{
SARCOIDOSIS WITH BONE AND SPINAL CORD INVOLVEMENT MIMICKING METASTATIC DISEASE
}

Leila Patrícia Muniz Veloso ${ }^{1, *}$, Claudia Lopes Santoro Neiva1, Lilian Santuza Santos Porto ${ }^{1}$, Fernanda Armond Castro ${ }^{1}$

1.Santa Casa de Belo Horizonte, Belo Horizonte (MG), Brazil.

*Corresponding author: leilapmuniz@hotmail.com

\section{BACKGROUND}

Sarcoidosis is a systemic disease that histologically presents non-caseous granuloma and, typically, bilateral hilar adenopathy, pulmonary infiltrate, skin and/or eye lesions. Bone and joint manifestations occur less commonly. Patients with widespread disease may have impairment of peripheral appendicular skeleton in 1-13\% of cases. Primary skeletal presentation is rare. Bone sarcoidosis occurs in approximately $5 \%$ of patients with sarcoidosis (range 1 to $13 \%$ ), although its frequency is likely to be underestimated, as it is usually asymptomatic. Likewise, sarcoidosis rarely affects the bone marrow, with possible hematological changes. It is more common in middle-aged women and afro-descendants. It usually appears later than many other common manifestations of sarcoidosis. The case below refers to sarcoidosis with lytic lesions disseminated in the axial skeleton and long bones, in addition to marked anemia, mimicking metastatic disease.

\section{CASE REPORT}

Female, brown, 65 years old, diabetic started bone pain in lower limbs and scapula with six months of evolution, associated with weight loss of approximately $18 \mathrm{~kg}$ and bilateral inguinal lymph node enlargement. He evolved with loss of renal function, proteinuria, anemia, and hypercalcemia (Cr: 3.43, Ur: 126, Hb: 6.7, proteinuria: 1060 mg/day, ionic calcium: 7.8). Radiography of skullcap was performed, showing lytic lesions. Initially, the hypothesis of multiple myeloma was raised, with protein electrophoresis that did not show a monoclonal peak, urinary immunofixation, also without monoclonal protein and myelogram without an increase in plasma series. Chest computed tomography (CT) scan showed bilateral hilar adenopathy, scintigraphy with disseminated bone involvement in the spine, hips, ribs, skull and long bones, such as femur and humerus, compatible with sarcoidosis, but cannot rule out metastatic disease. Bone marrow biopsy with granulomatous myelitis with giant cells, in addition to lymph node biopsy with granulomatous lymphadenitis with giant cells, closing the diagnosis for sarcoidosis with bone and medullary involvement by bone marrow infiltration.

\section{CONCLUSION}

Sarcoidosis can affect the musculoskeletal system and bone marrow, although it is quite rare. It is a challenging diagnosis and the diagnosis of malignant diseases should be excluded initially. 